\title{
Assessment of apoptosis, MMP-1, MMP-3, TIMP-2 expression and mechanical and biochemical properties of fresh rabbit's medial meniscus stored two weeks under tissue culture conditions
}

Tomasz J. Zwierzchowski ${ }^{1}$, Olga Stasikowska-Kanicka², Jolanta Janus³, Włodzimierz Konecki, Marian Danilewicz ${ }^{2}$, Jarosław Fabiś ${ }^{1}$

\author{
${ }^{1}$ Orthopaedic Department, Arthroscopy and Sports Traumatology Clinic, \\ Medical University of Lodz, Poland \\ 2Department of Nephropathology, Medical University of Lodz, Poland \\ ${ }^{3}$ Department of Pathophysiology, Medical University of Lodz, Poland \\ ${ }^{4}$ Department of Fibre Physics and Textile Metrology, Technical University of Lodz, \\ Poland
}

Submitted: 30 May 2013

Accepted: 10 August 2013

Arch Med Sci 2014; 10, 1: 167-173

DOI: 10.5114 /aoms.2014.40276

Copyright (c) 2014 Termedia \& Banach

\section{Abstract}

Introduction: The purpose of this study was to assess apoptosis, the expression of MMP-1, MMP-3 and TIMP-2, as well as the mechanical and biochemical properties of fresh rabbit medial meniscus after 2 weeks stored under tissue culture conditions.

Material and methods: The study material included 26 rabbit's medial menisci. Fourteen menisci were stored for 2 weeks under tissue culture conditions. Thirteen menisci were subjected to immunohistochemistry tests. Apoptosis (TUNEL method) and the expression of MMP-1 (collagenase-1), MMP-3 (stromelysin-2) and TIMP-2 (tissue inhibitor of metalloproteinases-2) were estimated semiquantitatively. The remaining menisci were tested mechanically and biochemically. The mechanical properties were assessed by the degree of elasticity. Biochemical composition was based on the content analysis of water, total collagen and glycosaminoglycans.

Results: As in the control group, the conducted study did not reveal any apoptosis in the stored menisci. The study group showed a slight expression of MMP-1 $(0.27 \pm 0.19)$, MMP-3 $(0.30 \pm 0.20)$ and TIMP-2 $(0.33 \pm 0.20)-$ no statistically significant difference from controls. The degree of elasticity was $28.51 \pm 1.53$ in the study group, and this did not statistically differ from the control group. No significant biochemical differences were identified in any other monitored parameter.

Conclusions: The conducted in vitro study did not show any negative influence of a 2-week storage period under tissue culture conditions on the apoptosis and expression of MMP-1, MMP-3 and TIMP-2 in rabbit fresh menisci, nor any impact on their mechanical and biochemical properties.

Key words: apoptosis, metalloproteinases, viable meniscal transplant properties.

\section{Introduction}

Fresh menisci are claimed to be the best transplant material for allografts in humans [1-3]. The presence of viable cells and preserved

\author{
Corresponding author: \\ Tomasz J. Zwierzchowski PhD \\ Orthopedic Department \\ Arthroscopic Clinic \\ 113 Żeromskiego St \\ 90-549 Lodz, Poland \\ Phone: +48426361398, \\ +48509508781 \\ Fax: +48426393511 \\ E-mail: \\ zwierzchowski.jacek@ \\ gmail.com
}


structure at the moment of transplantation are believed to have a positive effect on the allograft integration processes and its final result [4].

Tissue culture conditions facilitate the storage of fresh menisci [5]. Verbruggen et al. demonstrated that fresh meniscal cells may be stored under tissue culture conditions for 2 weeks while maintaining their viability, and their ability to produce extracellular matrix elements [6]. Fresh menisci stored under tissue culture conditions are used in clinical practice, and Verdonk et al. call this kind of allograft "viable" [7].

Experimental conditions allow the use of an autograft which fits perfectly and which facilitates the objective measurement of changes both in the transplanted cartilage and the joint cartilage [8-10]. To elucidate the biological processes present in the transplanted meniscus that could have an impact on the biological properties of the graft, this study was undertaken with the approved and used rabbit model [11-15]. This study revealed disorders of homeostasis in the form of excessive apoptosis, as well as altered mechanical and biochemical properties in viable rabbit menisci autografts in a 6-month follow-up. In allografts, the changes have been found to be more profound and accompanied by increased expression of MMP-1 (collagenase-1), MMP-3 (stromelysin-1) and TIMP-2 (tissue inhibitor of metalloproteinases-2) [16]. However, the in vivo findings pose the question of whether the described unfavourable changes in the graft are induced as early as during storage. As no unequivocal answer could be found in the literature, the present in vitro study was carried out.

The hypothesis of the study was that storage under tissue culture conditions for 2 weeks would influence apoptosis, metalloproteinase expression, and biochemical and mechanical properties of the fresh menisci.

The aim of this work was to assess apoptosis, MMP-1, MMP-3 and TIMP-2 expression, as well as the mechanical and biochemical properties of the fresh rabbit medial menisci after a 2-week storage period under tissue culture conditions.

\section{Material and methods}

\section{Study design}

Twenty-six medial menisci, harvested from the right knee of New Zealand white male rabbits (bred together, 8 months old, weighing 3500$4000 \mathrm{~g}$ ) were used for the study after receiving the approval of the local Committee for Experiments on the Animals (number $七 / B D / 254$ ).

The animals were euthanised by administering a lethal dose of pentobarbital into the marginal ear vein. After removing the muscles, the joints were opened. Then the medial menisci were harvested with extreme caution. Immediately after harvesting, 14 fresh menisci were put into the tissue culture for 14 days and 12 fresh menisci were kept as a control group.

The 14 excised menisci were placed in a tissue culture flask (Nunc) with Eagle medium (DMEM: Ham F12 (1: 1), Gibco) with the addition of $10 \%$ fetal calf serum (Sigma) and 1\% penicillin, streptomycin and Fungizone (Gibco). The containers were stored in an incubator for 2 weeks (7 menisci), at $37^{\circ} \mathrm{C}$ in an atmosphere of $5 \% \mathrm{CO}_{2}$ and $95 \%$ air. Every 3 days, the bottles and medium were exchanged [5].

Thirteen menisci (7 from the study group and 6 from the control group) designated for immunohistochemical examinations were placed in containers with $10 \%$ buffered formalin. The remaining 13 menisci were immediately subjected to mechanical tests and analysis of biochemical composition. Each test was performed within the posterior third of the menisci [16].

\section{Apoptosis detection}

Apoptosis was evaluated using the TUNEL method. Formalin-fixed, paraffin-embedded tissue sections were mounted onto SuperFrost slides and deparaffinized with xylene, washed with alcohol and transferred to distilled water. Afterwards, a TUNEL Apoptosis Detection Kit (DNA Fragmentation/Fluorescence Staining; No 17-141; Upstate NY) was used according to the instructions of the manufacturer. In each specimen immunofluorescence was recorded semiquantitatively according to Durand et al. in 3-7 adjacent high power fields and the mean grade was calculated [17].

\section{Immunohistochemistry}

Paraffin sections were mounted onto SuperFrost slides, deparaffinized, then treated in a microwave oven in a solution of EDTA, $\mathrm{pH} 8.0$ for $20 \min (2 \times 360 \mathrm{~W}-5 \mathrm{~min}, 2 \times 180 \mathrm{~W}-5 \mathrm{~min})$ and transferred to distilled water. Endogenous peroxidase activity was blocked by $0.3 \%$ hydrogen peroxide in distilled water for $30 \mathrm{~min}$, and then the sections were rinsed with Tris-buffered saline (TBS, DakoCytomation, Denmark) and incubated overnight at $4^{\circ} \mathrm{C}$, with the following mouse monoclonal antibodies: anti-MMP-1 (Chemicon, MAB 3307, Germany, dilution $1: 200$ ), anti-MMP-3 (Chemicon, MAB 3312, Germany, dilution $1: 250)$ and rabbit monoclonal antibody against TIMP-2 (Chemicon, AB 19029, Germany, dilution 1 : 300). Afterwards, appropriate EnVision + Systems-HRP (DakoCytomation, Denmark) were used, prepared according to the instructions of the manufacturer. Visualisation was performed by incubating the 
sections in a solution of 3,3'-diaminobenzidine (DakoCytomation, Denmark). After washing, the sections were counter-stained with haematoxylin and cover-slipped. For each antibody and for each sample, negative controls were processed by incubation in the absence of the primary antibody, and were found to always yield negative results.

The staining intensities of MMP-1, MMP-3 and TIMP-2 were recorded semiquantitatively according to Choi et al. in 3-7 consecutive high power fields [18]. Then the mean grade was calculated.

Apoptosis and immunohistochemistry evaluation was performed by a single observer, who was blinded to the experimental groups.

\section{Mechanical test}

The mechanical evaluation of menisci was based on a stress-relaxation test [19]. Immediately after harvesting ( 6 fresh menisci) or incubation (7 menisci) the posterior thirds of menisci were cut with a sterile surgical blade. The cut menisci probes were weighed and subjected to a compression test on a floor model INSTRON TT-BM testing instrument. The meniscus probe was placed in a specially designed metal nonporous matrix with a shape matching the concavo-cuneiform shape of the meniscus, which prevented the specimen from shifting laterally during compression. The posterior region of the menisci was compressed with a force of $2.5 \mathrm{~N}$, held for 5 min and allowed to relax. The degree of elasticity, calculated from elastic and total elongation, was determined from the obtained hysteresis curve [16]. The mechanical test's parameters were adjusted on the basis of rabbit's medial meniscus mechanical properties proposed by Sommerlath and Gilquist [15].

\section{Biochemical evaluation}

Directly after mechanical testing, the menisci probes were dried, weighed and subjected to biochemical analysis. The water content was calculated by subtracting the dry weight from the wet weight of the meniscus. The colorimetric method described by Woessner was used to assess the hydroxyproline fraction [20]. The total collagen content was derived from hydroxyproline by multiplying its value by the factor of 7.4 suggested by Ingman et al. [21]. The determination of total sulphated GAG content was based on the method described by Fernale et al. [22].

\section{Statistical analysis}

All values were expressed as the mean \pm SD (standard deviation). All the variables were treated as measurable ones. The Shapiro-Wilk normality test served as a basis for deciding on a parametric or non-parametric statistical test. In all groups there was no reason to dismiss the hypothesis that the variables have a normal distribution, except for apoptosis, which does not appear in the control group. The Shapiro-Wilk normality test results enabled the application of parametric methods in the subsequent calculations. Next Levene's test was conducted, which rejected the hypothesis of homogeneity of variance for apoptosis and immunohistochemistry. The Mann-Whitney test was used for the evaluation of statistical differences between research and control groups. Value of $p<0.05$ was considered to be the level of statistical significance.

\section{Results}

\section{Apoptosis evaluation}

As in the control menisci, no apoptosis of meniscal cells was identified in the study group by the TUNEL method (Figures $1 \mathrm{~A}$ and $2 \mathrm{~A}$ ).

\section{Immunohistochemistry}

Both in the study group and the control group, slight expression of MMP-1, MMP-3 and TIMP-2 was observed (Figures 1 B-D, 2 B-D). No statistically significant differences were found between the groups. The detailed values of metalloproteinase expression are presented in Table I.

\section{Mechanical testing}

No significant differences were observed between the elasticity values for the control and study groups. The detailed elasticity values are provided in Table II.

\section{Biochemical analysis}

The statistical analysis did not reveal significant differences between the control and study groups in any of the three parameters: water, glycosaminoglycans and total collagen content. The detailed values are given in Table II.

\section{Discussion}

A review of the literature indicates that no analysis has yet been performed on the influence of a 2-week period of storage under tissue culture conditions on apoptosis, tissue metalloproteinase expression and the mechanical properties of fresh menisci. The aim of this work was to assess apoptosis, MMP-1, MMP-3 and TIMP-2 expression, as well as the mechanical and biochemical properties of the fresh rabbit medial menisci after a 2-week storage period under tissue culture conditions. The hypothesis of the study was that storage under tissue culture conditions for 2 weeks would influence apoptosis, metalloproteinase expression, 

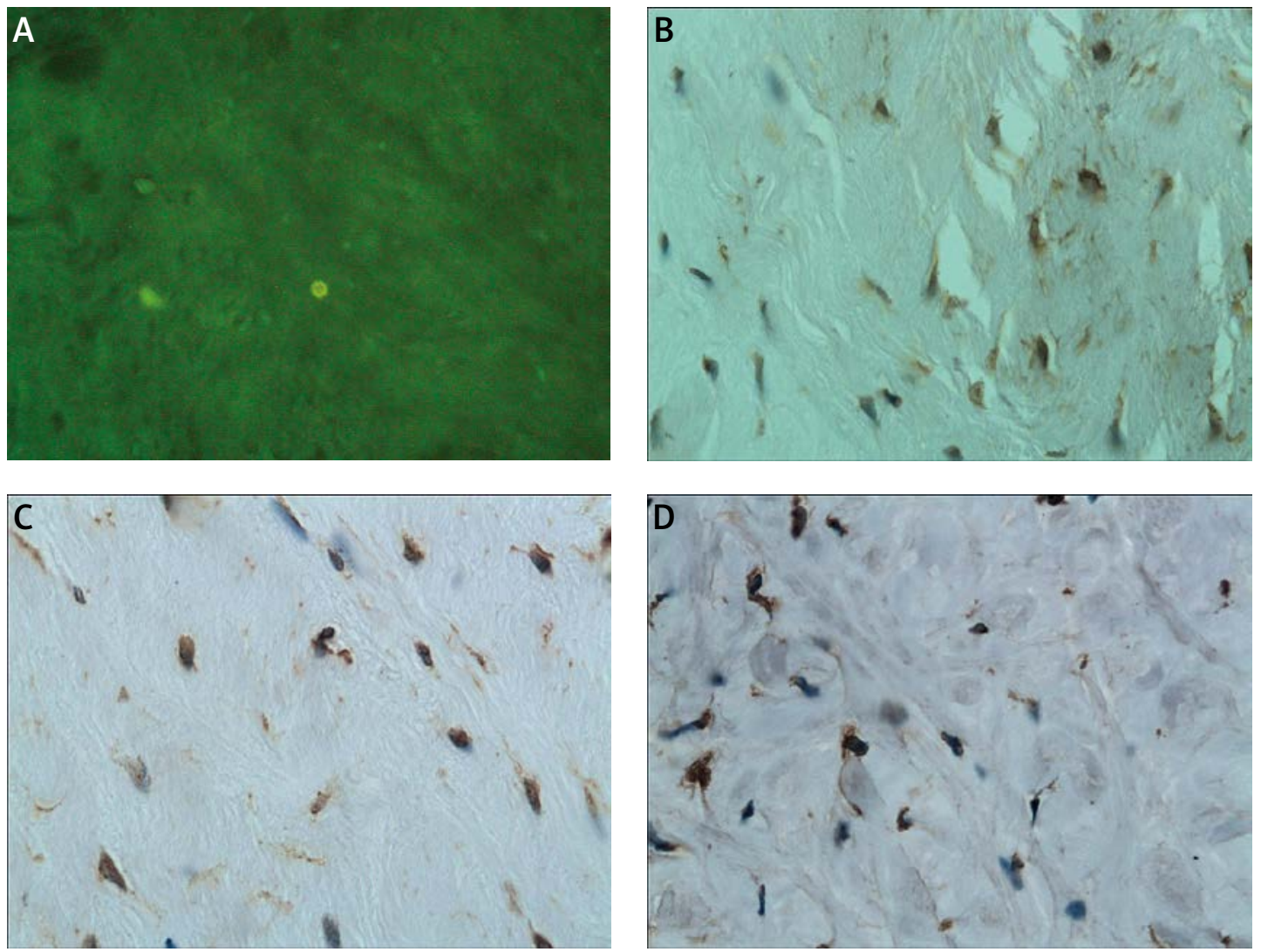

Figure 1. Posterior part of the fresh meniscus (control): A - TUNEL staining demonstrating lack of apoptotic cells (JPEG) magn. 200x, B - Immunohistochemical staining of MMP-1 (JPEG) magn. 400×, C - Immunohistochemical staining of MMP-3 (JPEG) magn. 400x, D - Immunohistochemical staining of TIMP-2 (JPEG) magn. 400x

and biochemical and mechanical properties of the fresh menisci.

The TUNEL method in the present study confirmed that apoptosis was not present in either the stored menisci or the menisci from the control group. Apoptosis is a highly regulated form of cell death, which allows the elimination of individual worn-out or mutated cells [23-25]. Although meniscal cells are subjected to apoptosis, it can hardly ever be observed under physiological conditions $[11,26]$, which confirms our findings on menisci from the control group. The absence of apoptosis in the control group was also described in an earlier work [16]. Apoptosis is clinically significant and its dysregulation, which occurs in meniscus homeostatic disorders, is an indicator of a degenerative process [11]. Research using experimental models of the degenerative disease of menisci shows that excessive apoptosis is typical of the early stage of degeneration, it is the only indicator of degeneration and it is revealed 3 weeks after the induced degenerative process $[13,27]$. The present in vitro studies were conducted in a similar period, i.e. in a 2-week follow-up incubation. Despite using a different incubation temperature $\left(4^{\circ} \mathrm{C}\right)$ than the present study, Ball et al. showed that the viability of osteochondral grafts do not change within a 14-day storage period under tissue culture conditions [28]. Verbruggen et al. claim that the viability of stored meniscal cells should be maintained through their ability to produce matrix particles [6]. Le Graverand et al. proved that excessive apoptosis leads to a decrease in the cell population in the degenerated meniscus [13]. The lack of apoptosis in the study group indicates that a 2-week storage period under tissue culture conditions does not have a negative impact on the apoptosis of fresh menisci, has no influence on cell number and confirms that homeostatic disorders do not exist. The statistically significant intensification of apoptosis in both auto- and allografts of fresh meniscus given above, as well as the lack of apoptosis in stored menisci identified in the present study, confirm that disorders of apoptosis occur after transplantation [16].

The conducted mechanical analysis of the stored menisci did not reveal significant differences from the control menisci, which implies that mechanical properties of the stored menisci are not influenced. Kwan et al. performed a similar study on the mechanical properties of the articular surface in dogs [29]. They confirmed that 28 days of tissue culture conditions do not change the mechanical properties of the tibia plateau. 

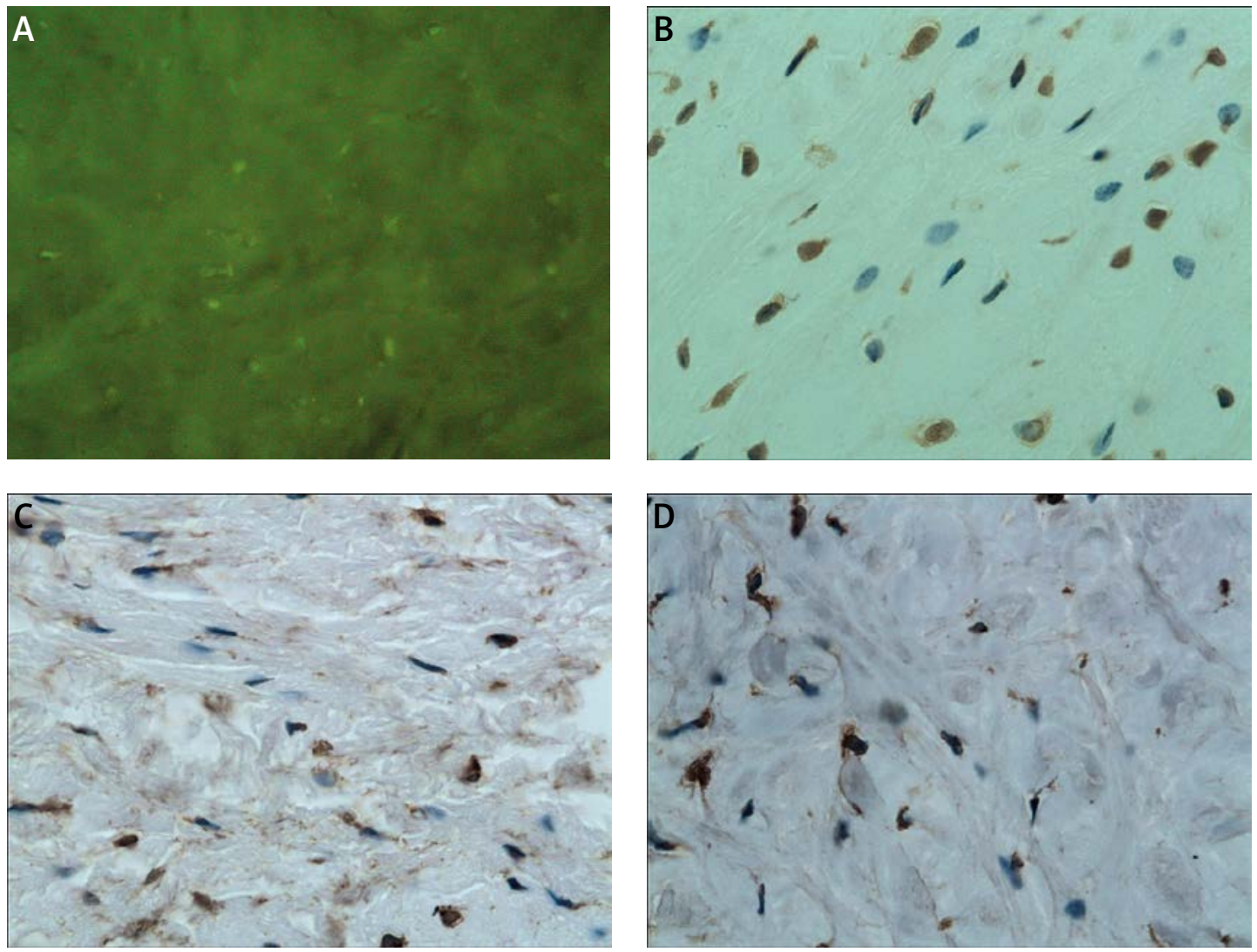

Figure 2. Posterior part of the fresh meniscus after 2-week storage under tissue conditions (study group): A - TUNEL staining demonstrating lack of apoptotic cells (JPEG) magn. 200×, B - Immunohistochemical staining of MMP-1 (JPEG) magn. 400×, C - Immunohistochemical staining of MMP-3 (JPEG) magn. 400x, D - Immunohistochemical staining of TIMP-2 (JPEG) magn. 400x

Table I. Immunoexpression of apoptosis, MMP-1, MMP-3 and TIMP-2 in the control group and in the study group

\begin{tabular}{|lcccc|}
\hline Group & Apoptosis & $\begin{array}{c}\text { MMP-1 } \\
\text { (collagenase-1) }\end{array}$ & $\begin{array}{c}\text { MMP-3 } \\
\text { (stromelysin-1) }\end{array}$ & $\begin{array}{c}\text { TIMP-2 } \\
\text { (tissue inhibitor of } \\
\text { metalloproteinases-2) }\end{array}$ \\
\hline Control & $0.00 \pm 0.00$ & $0.15 \pm 0.15$ & $0.20 \pm 0.03$ & $0.22 \pm 0.06$ \\
\hline Study & $0.00 \pm 0.00$ & $0.27 \pm 0.19$ & $0.30 \pm 0.20$ & $0.33 \pm 0.20$ \\
\hline Value of $p$ & & $0.27^{*}$ & $0.24^{*}$ & $0.22^{*}$ \\
\hline
\end{tabular}

Mean value \pm standard deviation (SD), *value of $p-N S$

Table II. Value of degree of elasticity, water, total collagen and GAGs content in the study group and in the control group

\begin{tabular}{|lcccc|}
\hline Group & Degree of elasticity & Water $(\%)$ & $\begin{array}{c}\text { Total collagen } \\
{[\mu \mathrm{g} / \mathrm{mg} \text { dry mass }]}\end{array}$ & $\begin{array}{c}\text { GAGs } \\
\text { (glycosaminoglycans) } \\
{[\mu \mathrm{g} / \mathrm{mg} \text { dry mass }]}\end{array}$ \\
\hline Control & $29.48 \pm 1.05$ & $73.25 \pm 1.07$ & $819.85 \pm 9.77$ & $94.95 \pm 2.84$ \\
\hline Study & $28.51 \pm 1.53$ & $74.25 \pm 1.38$ & $810.52 \pm 7.18$ & $94.19 \pm 2.49$ \\
\hline Value of $p$ & $0.97^{*}$ & $0.18^{*}$ & $0.07^{*}$ & $0.62^{*}$ \\
\hline
\end{tabular}

Mean value \pm standard deviation (SD), *Value of $p-N S$

The influence of tissue culture conditions on mechanical properties of osteochondral allografts was studied by Wayne et al., who did not find any significant impact of incubation [30]; however, the storage temperature in their work was $4^{\circ} \mathrm{C}[29$, 30]. As altered mechanical properties foster meniscal damage and correlate with excessive apoptosis $[12,13]$, the unchanged mechanical proper- 
ties found in the stored menisci confirm the lack of apoptosis in these menisci.

Parallel findings were obtained from the biochemical analysis of the stored menisci. The lack of any significant difference between the study and control groups with regard to water, total collagen and glycosaminoglycan content shows that storage method has no influence on the biochemical properties of the menisci. Amiel et al. note that tissue culture conditions do not change the biochemical contents of the hyaline cartilage [31], while Ball et al. demonstrate that 2-week storage under tissue culture conditions does not change glycosaminoglycan content in osteochondral allografts [28]. Le Graverand et al. related apoptosis to the change in the biochemical contents of menisci $[12,13]$. The unchanged biochemical properties of the stored menisci found in the present study confirm previous findings on apoptosis. The present results suggest that changes in both biochemical content and mechanical properties occur after the implantation of menisci rather than during their storage [16].

Immunohistochemical tests showed slight expression of collagenase- 1 and stromelysin- 1 in the stored menisci, although this was not significantly different from the control menisci. The MMP-1 (collagenase-1) and MMP-3 (stromelysin-1) are the main effectors of changes in the extracellular matrix of menisci $[12,26,32]$. The present results indicate that changes in the extracellular matrix of the stored menisci occur at the physiological level $[33,34]$. Metalloproteinase expression increases in profound disorders of homeostasis such as degenerations [12, 32]. The obtained re sults of MMP-1 and MMP-3 expression show that disorders of homeostasis do not occur in the stored menisci. Not only does MMP-3 take part in the degradation of proteoglycans, which allows the lysis of collagen via collagenase, as reported by Tsuzaki et al., but it also participates in the alternative method of pro-MMP-9 activation as described by Dreier et al. [35, 36]. Witty et al. confirm that overexpression of MMP-3 can induce apoptosis [37]. The low, statistically insignificant, level of TIMP-2 expression seen in the study group confirms that physiological changes occurred in the matrix and also that no disorders were present in homeostasis. Apart from hampering the broad spectrum of metalloproteinase, TIMP-2 also manifests a number of biological activities. It regulates apoptosis, influences cell proliferation and activates pro-MMP-2 $[38,39]$. The present results show that the high expression of MMP-1, MMP-3 and TIMP-2 described in the allograft group occurs after transplantation [16]. Hashimoto et al. demonstrated that disturbed apoptosis is related to increased metalloproteinase expression [11]. Consequently, the low level of metalloproteinase expression seen in the study group is related to the lack of apoptosis in the stored menisci [39].

The studies are limited as the obtained in vitro results refer only to the menisci of rabbits, which were further limited to posterior thirds of the menisci. Moreover, immunohistochemical tests were limited to the selected metalloproteinases and the methodology of mechanical analysis was simplified. Longer storage times of over a fortnight were not investigated [16]. We performed no power analysis or sample size calculation.

The conducted in vitro studies, which were taken up after earlier research, show that 2-week storage under tissue culture conditions does not have a negative influence on apoptosis, the expression of metalloproteinases, or the homeostasis of fresh menisci, nor does it change their mechanical and biochemical properties. The intensification of apoptosis, the increase of metalloproteinase expression and the change of mechanical and biochemical properties have been shown to occur after the implantation of fresh menisci, not while they are stored under tissue culture conditions.

\section{Acknowledgments}

This study was supported by a research project of the Medical University of Lodz: 502-17-263.

\section{References}

1. Felix NA, Paulos LE. Current status of meniscal transplantation. Knee 2003; 10: 13-7.

2. Rijk P. Meniscal allograft transplantation - part I: background, results, graft selection and preservation and surgical consideration. Arthroscopy 2004; 20: 728-43.

3. Siegel MG, Robert CS. Meniscal allografts. Clin Sports Med 1993; 12: 59-80.

4. Verdonk R. Alternative treatments for meniscal injuries. J Bone Joint Surg Br 1997; 79: 866-73.

5. Verdonk PCM, Demurie A, Almqvist KF, Veys EM, Verbruggen G, Verdonk R. Transplantation of viable meniscal allograft. J Bone Joint Surg Am 2006; 88 (Suppl. 1.): 109-18.

6. Verbruggen G, Verdonk R, Veys EM, et al. Human meniscal proteoglycan metabolism in log-term tissue culture. Knee Surg Sports Traumatol Arthrosc 1996; 4: 57-63.

7. Verdonk PCM, Demurie A, Almqvist KF, Veys EM, Verbruggen G, Verdonk R. Transplantation of viable meniscal allograft. Survivorship analysis and clinical outcome of one hundred cases. J Bone Joint Surg Am 2005; 87: 715-24.

8. Stone KR, Rodkey WG, Kinney LA, Steadman JR. Autogenous replacement of the meniscus cartilage: analysis of results and mechanisms of failure. Arthroscopy 1995; 11: 395-400.

9. Szomor ZL, Martin TE, Bonar F, Murrel GAC. The protecive effects of meniscal transplantation an cartilage. An experimental study in sheep. J Bone Joint Surg Am 2000; 8: 80-8.

10. Zwierzchowski TJ, Stasikowska-Kanicka O, Danilewicz M, Fabiś J. Assessment of apoptosis and MMP-1, MMP-3 
and TIMP-2 expression in tibial cartilage after viable medial meniscus transplantation in the rabbit. Arch Med Sci 2012; 8: 1108-14.

11. Hashimoto S, Takahashi K, Ochs RL, Coutts RD, Amiel D, Lotz M. Nitric oxide production and apoptosis in cells of the meniscus during experimental osteoarthritis. Arthritis Rheum 1999; 42: 2123-31.

12. Le Graverand HMP, Vegnon E, Otterness IG, Hart DA Early changes in lapine menisci during osteoarthritis development. Part I: cellular and matrix alteration. Osteoarthritis Cartilage2001; 9: 56-64.

13. Le Graverand HMP, Vegnon E, Otterness IG, Hart DA Early changes in lapine menisciduring osteoarthritis development. Part II: mollecularalteration. Osteoarthritis Cartilage 2001; 9: 65-72.

14. Rijk PC, Van Noorden C. Structural analysis of meniscal allografts after immediate and delayed transplantation in rabbits. Arthroscopy 2002; 18: 995-1001.

15. Sommerlath K, Gilquist J. The effect of an artificial meniscus substitute in a knee joint with resected anterior cruciate ligament. An experimental study in rabbits. Clin Orthop 1993; 289: 276-84.

16. Zwierzchowski TJ, Stasikowska-Kanicka O, Janus J, Konecki W, Danilewicz M, Fabiś J. Evidence for apoptosis, MMP-1, MMP-3 and TIMP-2 expression and their efect on the mechanical and biochemical properties of fresh viable knee medial meniscal allografts and autografts in the rabbit. Arch Med Sci 2012; 8: 724-32.

17. Durand $E$, Scoazec A, Lafont $A$, et al. In vivo induction of endothelial apoptosis leads to vessel thrombosis and endothelial denudation. A clue to the understanding of the mechanisms of thromotic plaque erosion. Circulation 2004; 109: 2503-6.

18. Choi HR, Kondo S, Hirose K, Ishiguro N, Hasegawa $Y$, Iwata $\mathrm{H}$. Expression and enzymatic activity of MMP-2 during healing process of the acute supraspinatus tendon tear inrabbits. J Orthop Res 2002; 20: 927-33.

19. DeHaven KE. The role of the meniscus. In: Articular cartilage and knee joint function: basic science and arthroscopy. Ewing JW (ed.). Raven, New York 1990; 103-15.

20. Woessner JF Jr. The determination of hydroxyproline in tissue and protein samples containing small portions of this amino acid. Arch Biochem Biophys 1961; 93: 440-7.

21. Ingman AM, Ghosh P, Taylor TKF. Variation of collagenuos and non-collagenous proteins of human menisci with age and degeneration. Gerontologia 1974; 20: 212-23.

22. Fernale RW, Buttle DJ, Barrett AJ. Improved quantitation and discrimination of sulfated glycosaminoglycans by use dimethylmethylene blue. Biochem Biophys Acta 1986; 883: 173-7.

23. Majno G, Joris I. Apoptosis, oncosis and necrosis. An overview of cell death. Am J Pathol 1995; 146: 3-15.

24. Liang QL, Wang BR, Li ZY, Chen GQ, Zhow Y. Effect of TSLC1 gene on growth and apoptosis in human esophageal carcinoma Eca109 cells. Arch Med Sci 2012; 8: 987-92.

25. Wyllie AH, Kerr JF, Currie AR. Cell death: the significance of apoptosis. Int Rev Cytol 1980; 68: 251-306.

26. Benjamin M, Ralps JR. Biology of fibrocartilage cells. Int Rev Cytol 2004; 233: 1-45.

27. Robertson CM, Pennock AT, Harwood FL, Pomerleau BA, Allen RT, Amiel D. Characterization of pro-apoptotic and matrix - degenerative gene expression following induction of osteoarthritis in mature and aged rabbits. Osteoarthritis Cartilage 2006; 14: 471-6.
28. Ball ST, Amiel D, Williams SK, et al. The effect of storage on fresh human osteochondral allografts. Clin Orthop 2004; 418: 246-52.

29. Kwan MK, Wayne JS, Woo SL, Field FP, Hoover J, Meyers $M$. Histological and biomechanical assessment of articular cartilage from stored osteochondral shell allografts. J Orthop Res 1989; 7: 637-44.

30. Wayne JS, Amiel D, Kwan MK. Long-term storage effects on canine osteochondral allografts. Acta Orthop Scand 1990; 61: 539-45.

31. Amiel D, Harwood FL, Hoover JA, Meyers WH. A histological and biochemical assessment of the cartilage matrix obtained from in vitro storage of osteochondral allografts. Connect Tissue Res 1989; 23: 89-99.

32. Bluteau G, Conrozier T, Mathieu P, Vignon E, Herbage D, Mallein-Gerin F. Matrix metalloproteinase-1, -3, -13 and aggrecanase- 1 and 2 are differentially expressed in experimental osteoarthritis. Biochim Biopys Acta 2001; 1526: 147-58.

33. Nagase $H$, Visse R. Structure and function of matrix metalloproteinases and TIMPs. Cardiovasc Res 2006; 69: 562-73.

34. Woessner J Jr. Matrix metalloproteinases and inhibitors in connective tissue remodeling. FASEB J 1991; 5: 2145-54.

35. Tsuzaki M, Guyton G, Garret W, et al. IL-1 beta induced COX2, MMP-1,3 and 13, ADAMTS-4, IL-1 beta and II- 6 in human tendon cells. J Orthop Res 2003; 21: 256-64.

36. Dreier R, Grässel S, Fuchs S, Schaumburger J, Bruckner P. Pro-MMP-9 is a specific macrophage product and is activated by osteoarthritic chondrocytes via MMP-3 or MT1-MMP/MMP-13 cascade. Exp Cell Res 2004; 297 : 303-12.

37. Witty JP, Lempka T, Coffey Jr RJ, Matrisian LM. Decreased tumor formation in 7,12-dimethylbenzanthracene-treared stromielysin-1 transgenic mice is associated with alteration in mammary epithelial cell apoptosis. Cancer Res 1995; 55: 1401-6.

38. Baker AH, Edwards DR, Murphy G. Metalloproteinase inhibitors: biologic actions and therapeutic opportunities. J Cell Sci 2002; 115: 3719-27.

39. Jiang Y, Goldberg ID, Shi E. Complex roles of tissue inhibitors of metalloproteinases in cancer. Oncogene 2002; 21: 2245-52. 